\title{
Frontières
}

\section{Comprendre la douleur chronique pour éviter la douleur de l'incompréhension}

\section{Yvon Beauchamp}

Volume 17, numéro 2, printemps 2005

Surtout, ne pas souffrir

URI : https://id.erudit.org/iderudit/1073492ar

DOI : https://doi.org/10.7202/1073492ar

Aller au sommaire du numéro

Éditeur(s)

Université du Québec à Montréal

ISSN

1180-3479 (imprimé)

1916-0976 (numérique)

Découvrir la revue

Citer cet article

Beauchamp, Y. (2005). Comprendre la douleur chronique pour éviter la douleur de l'incompréhension. Frontières, 17(2), 71-76.

https://doi.org/10.7202/1073492ar
Résumé de l'article

La douleur chronique est présente quotidiennement dans toutes les sphères médicales. Les malades qui en sont atteints n'acceptent plus de souffrir et ne tolèrent plus que leurs soignants ignorent leurs douleurs. Souvent cette ignorance par les médecins du phénomène douloureux est le reflet de leur ignorance des mécanismes fondamentaux neuro-endocriniens de la douleur. Il est plus facile d'ignorer ou de nier l'existence de ce qu'on ne saurait expliquer. Malheureusement, comment et pourquoi apaiser ce qui ne pose pas problème pour certains médecins? 


\section{Résumé}

La douleur chronique est présente quotidiennement dans toutes les sphères médicales. Les malades qui en sont atteints n'acceptent plus de souffrir et ne tolèrent plus que leurs soignants ignorent leurs douleurs. Souvent cette ignorance par les médecins du phénomène douloureux est le reflet de leur ignorance des mécanismes fondamentaux neuro-endocriniens de la douleur. II est plus facile d'ignorer ou de nier l'existence de ce qu'on ne saurait expliquer. Malheureusement, comment et pourquoi apaiser ce qui ne pose pas problème pour certains médecins?

Mots clés : douleur chronique émotion - morphine - contrôle de la douleur.

\section{Abstract}

Pain is every where in medicine. Patients don't accept anymore to suffer and they don't tolerate that theirs physicians act like if they were unaware of their pains. Often this ignorance of the problem is the reflection of the ignorance of basic neurosciences and neuropharmacology. Why and how treat something we don't understand?

Keywords : chronic pain - emotion morphine - control of pain.

\section{Comprendre la douleur chronique pour éviter la douleur de l'incompréhension}

\section{«IL N'Y A QU'UNE SEULE DOULEUR QU'IL SOIT FACILE DE SUPPORTER, C'EST CELLE DES AUTRES. ॥}

RENÉ LERICHE, CHIRURGIEN

\section{Dr. Yvon Beauchamp, C.C.F.P., chef du service de soins palliatifs, Hôpital Sacré-Cœur de Montréal.}

Comment des malades avec des douleurs chroniques peuvent-ils trouver apaisement et soulagement alors que cette "maladie» est si peu connue, si peu acceptée et si peu traitée? Certains penseront que le terme «maladie» est inapproprié et abusif pour qualifier la douleur chronique. Pourtant tous les médecins qui ont à traiter les malades qui en sont affectés vous diront que c'est bien la perception qu'ils en ont, et tous les médecins impliqués en douleur chronique font la différence entre la douleur aiguë-symptôme et la douleur chronique-maladie (Melzack, 1989, p. 32). La douleur chronique se définit comme une maladie, c'est-à-dire comme une altération organique ou fonctionnelle considérée dans son évolution, et comme une entité définissable. Par comparaison, le symptôme douloureux aigu est plutôt de l'ordre du phénomène ou du caractère perceptible ou observable lié à un état qu'il permet de déceler (Robert, 1991, p. 1137 et 1905; Melzack et Wall, 1989, p. 32).

Une définition reconnue du terme «douleur» existe. Cette définition est celle d'un comité de travail de l'International
Association for the Study of Pain (IASP, 1979) et s'énonce comme: "une perception à la fois sensorielle et émotionnelle, désagréable, liée à une lésion tissulaire présente ou potentielle ou décrite en des termes impliquant une telle lésion» (Merskey et Bogduk, 1994, p. 209-214). Cette définition couvre un large champ et englobe des dimensions purement nociceptives mais aussi et surtout des éléments psychologiques qui définissent une dimension sensori-discriminative, une dimension affectivo-motivationnelle et une dimension cognitivo-évaluative (Melzack et Casey, 1968, p. 423-439).

La portion de la définition faisant appel à la perception de la sensation ne pose jamais problème. Ainsi, lorsqu'une personne se présente aux urgences avec une douleur à la poitrine, un électrocardiogramme perturbé et des résultats sanguins anormaux, le corps médical, l'employeur, les compagnies d'assurance et la famille croiront rapidement à la gravité de cette douleur aiguë nouvelle et facilement explicable. Même crédibilité si vous arrivez avec une fracture d'un membre; les gens, non seulement vous croiront, mais ils sympathiseront avec vous et tenteront de vous soulager; ils accepteront votre irritabilité, votre colère, votre anxiété, vos pleurs et vos gémissements. 
Avec les malades affligés de douleurs chroniques, il en est trop souvent autrement. Pourquoi? Est-ce que l'acuité et l'intensité d'une douleur soulèvent plus de compassion qu'une douleur tout aussi intense mais présente de plus longue date? Est-ce que l'acuité d'une douleur soulève plus de solidarité humaine que la chronicité qui amène un amenuisement du capital de compassion? Les soignants en viennent-ils à s'habituer à voir le même individu souffrir jour après jour? Y a-t-il un désinvestissement dans le désir d'aider ces gens ou bien ne s'agit-il pas simplement d'un sentiment graduel d'impuissance qui s'installe comme pour toutes les maladies chroniques?

\section{LA DOULEUR CHRONIQUE}

La douleur chronique obéit à des mécanismes électriques et chimiques différents de ceux de la douleur aiguë. Ces mécanismes devenus pathologiques à cause de l'étonnante plasticité du système nerveux périphérique et central bombardent le cerveau émotionnel de l'humain. Ce cerveau ancien situé sous le cortex et se définissant par des structures interreliées qui ont pour noms, amygdales cérébrales, hippocampe, gyrus cingulaire, corps mammilaires, est appelé "système limbique». Il est le substratum anatomique de l'élaboration de nos comportements émotionnels. Ce système, intégré aux fonctions supérieures de la pensée et de la mémoire, est aussi en relation avec notre système endocrinien qui défend l'organisme humain contre des agressions extérieures et qui est responsable du maintien de l'homéostasie interne.

Les phénomènes douloureux, surtout chroniques, font toujours appel à la globalité des systèmes de l'humain et ne vouloir traiter que des symptômes physiques est le meilleur chemin vers l'insuccès. C'est le plus souvent la portion émotionnelle de la douleur qui est occultée par le personnel soignant, tout en reconnaissant que le traitement de la partie purement sensorielle laisse beaucoup à désirer. En effet on tient très peu compte du fait que la douleur est aussi une émotion et que la douleur peut être réelle sans lésion apparente. L'exemple extrême que tous connaissent est la perception d'un membre amputé, ressenti et décrit comme un fantôme souvent douloureux. Donc, point n'est besoin d'un tissu lésé pour vivre une douleur. Il est possible de ressentir la douleur longtemps après qu'une guérison apparente et superficielle s'est produite. Il suffit de questionner une personne qui a fait un zona cutané mais dont la peau est tout à fait saine après guérison et qui décrira des chocs électriques et des brûlures continuelles qui perturbent son sommeil et ses activités quotidiennes; cet individu, souvent, pense à la mort comme moyen de soulagement. Même chose pour des diabétiques dont les nerfs périphériques ont perdu la faculté d'enregistrer les sensations habituelles et normales, en plus de déformer ces sensations pour en faire des perceptions douloureuses. Que dire des gens affligés de maladies neurologiques dégénératives comme la sclérose en plaques ou les accidents vasculaires cérébraux, aux prises avec des douleurs créées par le système nerveux lui-même. Finalement, comment ne pas mentionner les malades qui développent des syndromes douloureux régionaux complexes (anciennement dénommés algodystrophie) en relation avec un traumatisme souvent banal qui les laissera dysfonctionnels ou afonctionnels d'un ou de plusieurs membres. Pour ces gens, à une vie professionnelle souvent détruite et aux problèmes économiques qui s'ensuivent, viennent s'ajouter les luttes opiniâtres que leur livrent la Commission de la santé et de la sécurité du travail (CSST), la Société de l'assurance automobile du Québec (SAAQ) ou les compagnies d'assurance.

\section{LA DOULEUR}

\section{N'EST PAS UNE SCIENCE}

\section{QUI S'ENSEIGNE À L'UNIVERSITÉ.}

Maladie peu comprise, malades incompris: incompréhension des proches, incompréhension du corps médical, incompréhension des compagnies d'assurance et des organismes gouvernementaux ou paragouvernementaux.

\section{DE NOS RACINES À AUJOURD'HUI}

Plus la médecine évolue, plus les médecins semblent s'éloigner de l'humanisme dont devrait se nourrir leur pratique quotidienne. Il fut un temps où Aristote considérait la douleur comme une passion de l'âme, une émotion (Chapman, 1997, p. 189-199)! On parle maintenant de phénomène douloureux entraînant un rejet, une restriction jusqu'à la destruction de la réflexion et une atteinte du langage et de la communication, donc une atteinte des instruments de l'interaction humaine. La douleur influence de ce fait trop souvent une vie par la perte de l'objectivité et une analyse devenue purement subjective et égocentrique de sa condition humaine (Scarry,1985).

Depuis la création de l'IASP dans les années 1970, la définition de la douleur a évolué pour en arriver à une dualité dans l'unité; la douleur est toujours une sensation et une émotion. Sensation et émotion ne sont pas la conséquence de la douleur, mais bien son point de départ. La partie émotionnelle de la douleur n'est pas une conséquence de la sensation douloureuse; l'émotion est fondamentalement et partiellement une partie de l'expérience consciente douloureuse vécue par un être humain.

Nos maîtres anciens possédaient pour seules armes l'intuition, l'observation et le raisonnement. Ils ne pouvaient qu'élaborer des théories difficilement démontrables selon nos normes scientifiques contemporaines. N'a aujourd'hui de crédibilité que ce qui peut se démontrer hors de tout doute en éprouvette, sous microscope ou sur une image sophistiquée de résonance magnétique. Peut-on expliquer la douleur émotionnelle en laboratoire, en imagerie ou en éprouvette? Malheureusement non! L'humoriste Yvon Deschamps n'était pas loin de la vérité quand il disait: "On veut pas le savoir, on veut le voir.» Aujourd'hui, on voudrait bien savoir et on voudrait bien voir et c'est ce que permet maintenant l'imagerie médicale avec ses appareils sophistiqués à émission de positrons. À défaut d'expliquer les rouages intracérébraux moléculaires, on peut visualiser l'activité cérébrale enclenchée par la douleur et pointer du doigt les zones cérébrales impliquées dans la perception de la douleur. Il n'y a pas encore consensus en ce qui a trait à la dimension émotionnelle de la douleur, mais les neurophysiologistes, neuropsychologues et neuroendocrinologues apportent beaucoup de nouvelles connaissances fondamentales au regard des substrats anatomiques, chimiques, électriques et organisationnels des faisceaux de la douleur.

\section{PREMIER PROBLÈME:}

\section{LA DÉFINITION DE LA DÉFINITION}

Ainsi, la douleur serait à la fois sensation et émotion; quelle serait donc la définition de l'émotion maintenant? Nous ne pouvons sûrement pas nous contenter de la définition et de l'intuition de nos maîtres grecs et simplement dire que l'émotion est une passion de l'âme car ceci soulève d'emblée la question de la nature de l'âme. Par ailleurs, mettre en évidence les structures physiques anatomiques, histologiques et électriques qui sous-tendent l'émotion, ne nous donne pas davantage une définition de la partie émotionnelle de la douleur mais au moins nous la situe dans l'univers du système nerveux central.

Pour Rolls (1986, p. 125-144), les émotions pourraient se ramener à des états provoqués par des stimuli intenses. On peut aller plus loin et comme Fonberg (1986, p. 301-331), penser que l'émotion est un processus nerveux qui détermine quels sont les stimuli environnementaux souhaitables pour l'organisme, fussent-ils positifs ou négatifs. En suivant cette piste directrice, on peut arriver à penser que l'émotion 


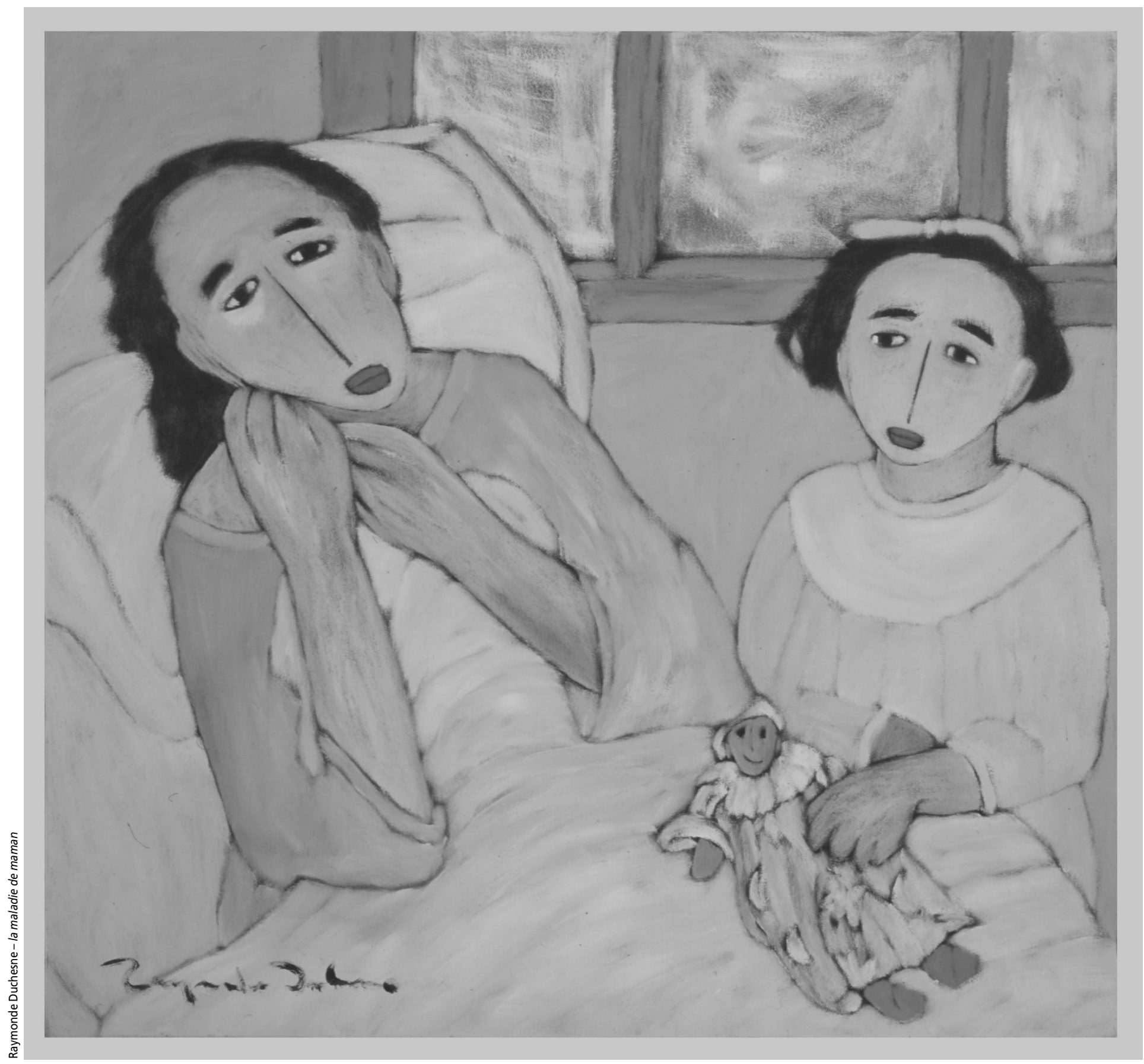

telle qu'elle est appréhendée en science fondamentale est finalement un mécanisme de défense qui a pour fonction le maintien d'une intégrité physique individuelle vis-à-vis l'environnement ainsi que la défense d'une intégrité individuelle interne par le maintien de l'homéostasie. La douleur est une menace pour l'individu et tant qu'elle est perçue comme telle, lorsqu'elle perdure plus longtemps que les délais habituels normaux de guérison, elle provoque des comportements tant volontaires qu'émotionnels.

La douleur immédiatement aiguë ressentie à la suite d'un traumatisme physique est plus de l'ordre du symptôme et elle est perçue

\section{le chant de sa plainte}

\section{s'est envolé}

\section{à l'effleurement du printemps}

comme un processus d'avertissement ayant pour but la protection d'une partie lésée afin d'en permettre la guérison. Cette douleur a un sens positif, bien que désagréable et elle est acceptable. Au contraire, la douleur chronique a perdu ce sens d'avertissement et de guérison; elle devient plutôt une maladie avec une panoplie de syndromes qui accaparent souvent tout le champ de la conscience en empêchant les échanges relationnels avec autrui.

\section{QUELLE ÉMOTION?}

Plutchick envisage pour l'humain des émotions qui ont des valeurs positives ou négatives. Ainsi on pourrait envisager des émotions primaires négatives qui seraient la peur, la colère, la tristesse et le dégoût (Plutchick, 1980, p. 3-33). En corollaire existeraient des émotions à valeur positive comme la joie, l'acceptation, l'espérance et la surprise. Chacune de ces émotions amène un comportement; ainsi la peur se traduira par une fuite, la colère par une attaque, la tristesse par les pleurs et le dégoût par le rejet. En contrepartie, la joie dictera un comportement de rencontre, l'acceptation favorisera la formation, l'espérance 
amènera l'estimation et la surprise, l'arrêt. Chacune de ces émotions, en favorisant ou en dictant un comportement, favorisera aussi l'atteinte d'une fonction sociobiologique qui, bien souvent, transcende l'individu. Celui qui a peur fuit, favorisant une fonction de mise hors d'atteinte, le colérique attaque favorisant une fonction de destruction, la personne triste pleure dans un but de réinsertion sociale et de demande d'aide, alors que celui qui a du dégoût vomit dans un but de rejet.

Aucune de ces émotions négatives ne correspond exactement à la douleur. Possiblement ou probablement que la peur est l'émotion qui se rapproche le plus de la douleur chronique, tout en conservant l'idée que l'émotion de la douleur évolue dans le temps et peut souvent devenir un amalgame de plusieurs émotions. Envisagés sous cet angle, les phénomènes émotionnels favoriseraient la survie de l'individu et de l'espèce et les réponses aux stimuli à travers les manifestations émotives favoriseraient l'adaptation biologique. Il est loisible de penser que l'émotion attribue une valeur positive (agréable) ou négative (désagréable) au stimulus en fonction de l'importance et de la signification biologique.

Grâce à la recherche fondamentale (neurologie, psychologie, endocrinologie, psychiatrie, pharmacologie, imagerie médicale avancée), on sait que le système limbique est le substratum anatomique de la partie émotionnelle de la douleur dans le système nerveux central. Ce système intégré est en lien étroit avec le système moteur, le système endocrinien, le système de la vigilance, du comportement, de la mémoire et de la cognition. C'est à la fois un système électrique et chimique de convergence, de passage et un point de départ. On pourrait résumer en disant que la douleur chronique est un état dépendant fondamentalement de la conscience d'une lésion tissulaire actuelle ou potentielle ou décrite en des termes impliquant une lésion mais aussi dépendant de phénomènes d'adaptation physique, émotionnelle et sociobiologique s'y rapportant. La pensée détermine l'émotion-sensation et l'émotion-sensation détermine la pensée (Chapman, 1997, p. 189-199). Le degré d'importance émotionnelle d'une douleur représente donc la frayeur que l'individu associe à l'événement déclencheur et traduit, en plus de l'exprimer, ce qu'il perçoit comme une menace à son intégrité biologique.

La portion émotionnelle de la douleur chronique me semble plus importante que la portion sensorielle lorsqu'il s'agit de faire une évaluation adéquate en clinique. Ce n'est pas tant l'intensité sensorielle du stimulus que sa charge émotionnelle qui est importante. Cette charge émotionnelle détermine le degré de désagrément filtré ou amplifié par l'expérience de vie de l'individu, sa mémoire, sa culture, ses angoisses du moment et le sens accordé à la douleur. Tous ces modulateurs font l'individu; si cet individu a des frayeurs incontrôlées de la douleur, un sentiment d'impuissance devant sa douleur et un sentiment dramatique de catastrophe, la guérison n'en sera que plus ardue. Comment «refaire» un individu et lui redonner confiance en lui-même? Comment lui faire accepter sa condition et faire naître en lui le désir d'inventer des stratégies pour penser à une nouvelle façon d'être et de vivre?

C'est à ce moment que commencent les difficultés du soignant, car il n'y a pas de test ou de radiographie, pour départager les émotions et pour savoir si le malade décrit bien ce qu'il ressent. Nous sommes comme Aristote; il nous faut revenir à l'observation, à la patience, à la communication et à l'écoute empathique. Nous avons toutefois des avantages puisque nous commençons à percevoir la mécanique électrique et chimique de l'émotion. Nous avons aussi des stratégies pharmacologiques, physiques, anesthésiques et psychologiques qui s'ajoutent au fur et à mesure de l'émergence des connaissances fondamentales.

\section{PEU DE CONNAISSANCES, PEU DE SOIGNANTS}

Pourquoi alors les gens souffrant de douleurs chroniques trouvent-ils si peu de réconfort, si peu de ressources médicales? La première réponse qui vient à l'esprit a trait au peu de formation, pour ne pas dire à l'absence de formation au premier cycle universitaire. La douleur n'est pas une science qui s'enseigne à l'université. Nos futurs médecins arrivent sans connaissances fondamentales de la neurologie et de la chimie de la douleur. Les étudiants sont intégrés dans les stages cliniques spécialisés ou de médecine familiale sans bagage scientifique théorique au regard de la douleur. Souvent leurs maîtres de stage sont tout aussi démunis qu'eux dans la pratique quotidienne du soulagement de la douleur. Souvent aussi pour ces professeurs de clinique la douleur n'est pas un enjeu puisque cela ne leur a jamais été présenté comme tel. On ne peut pas enseigner ce que l'on ne connaît pas.

Lignorance, si elle reste neutre, est sûrement un moindre mal comparativement à l'ignorance active et activiste qui propage souvent des notions erronées et des préjugés tenaces et archaïques. Qui dit douleur chronique dit traitement chronique, car la guérison est rarement une possibilité. Or dans la pharmacopée actuelle, on peut faire appel à certaines classes de médicaments de la famille des opioïdes et des cannabinoïdes. On se heurte déjà aux préjugés légaux et sociaux; préjugés repris et souvent enseignés par les médecins-professeurs à leurs étudiants. L'un de ces préjugés tenaces entretient le mythe de la dépendance psychologique quasi obligatoire pour un malade qui prendrait de la morphine ou l'un de ses dérivés. La littérature médicale et spécialisée en toxicologie est pourtant éloquente sur ce sujet; la prévalence dans la population est de 5 à 10\% pour le danger de devenir toxicomane avec l'emploi de morphine à des fins médicales et non récréatives (Robinson et al., 2001, p. 220-228; Chabal et Erjavec, 1997, p. 150-155). C'est dire que ces gens soutiennent par ignorance la thèse voulant que l'on s'abstienne de traiter avec des analgésiques puissants par peur qu'un malade sur dix ou sur vingt devienne dépendant psychologiquement. Que fait-on dans ce raisonnement des 95\% qui ne deviendraient pas dépendants et qui auraient vu leurs souffrances diminuer? Devrontils continuer à supporter leur fardeau et voir leur qualité de vie souvent déplorable se dégrader encore plus? Il existe pourtant des questionnaires validés qui nous permettent de détecter les gens qui sont à risque de développer une telle dépendance; nous pouvons ainsi encadrer médicalement, avec l'aide du pharmacien, ces malades qui doivent être aidés.

Une fois ces préjugés et ces craintes de bon aloi dédramatisés, il demeure que les médecins sont souvent peu enclins à utiliser ces classes de médicaments. Pourquoi?

Une raison invoquée le plus souvent est la crainte de se faire pointer du doigt par le Collège des médecins du Québec et d'avoir à justifier une pratique que l'on a déjà stigmatisée et surveillée. Encore là, ces craintes, qui ne sont souvent que prétexte pour ne pas s'occuper de cette clientèle très lourde, sont devenues depuis une dizaine d'années de faux problèmes. En effet le Collège a montré beaucoup d'ouverture d'esprit et un désir évident de faciliter le traitement des patients douloureux chroniques. Les

\section{FORMER UN MÉDECIN AU POINT DE VUE SCIENTIFIQUE}

DEMANDE ENTRE 7 ET 10 ANS, ALORS QUE FORMER UN MÉDECIN AU POINT DE VUE HUMAIN PEUT ÊTRE BEAUCOUP PLUS LONG! 


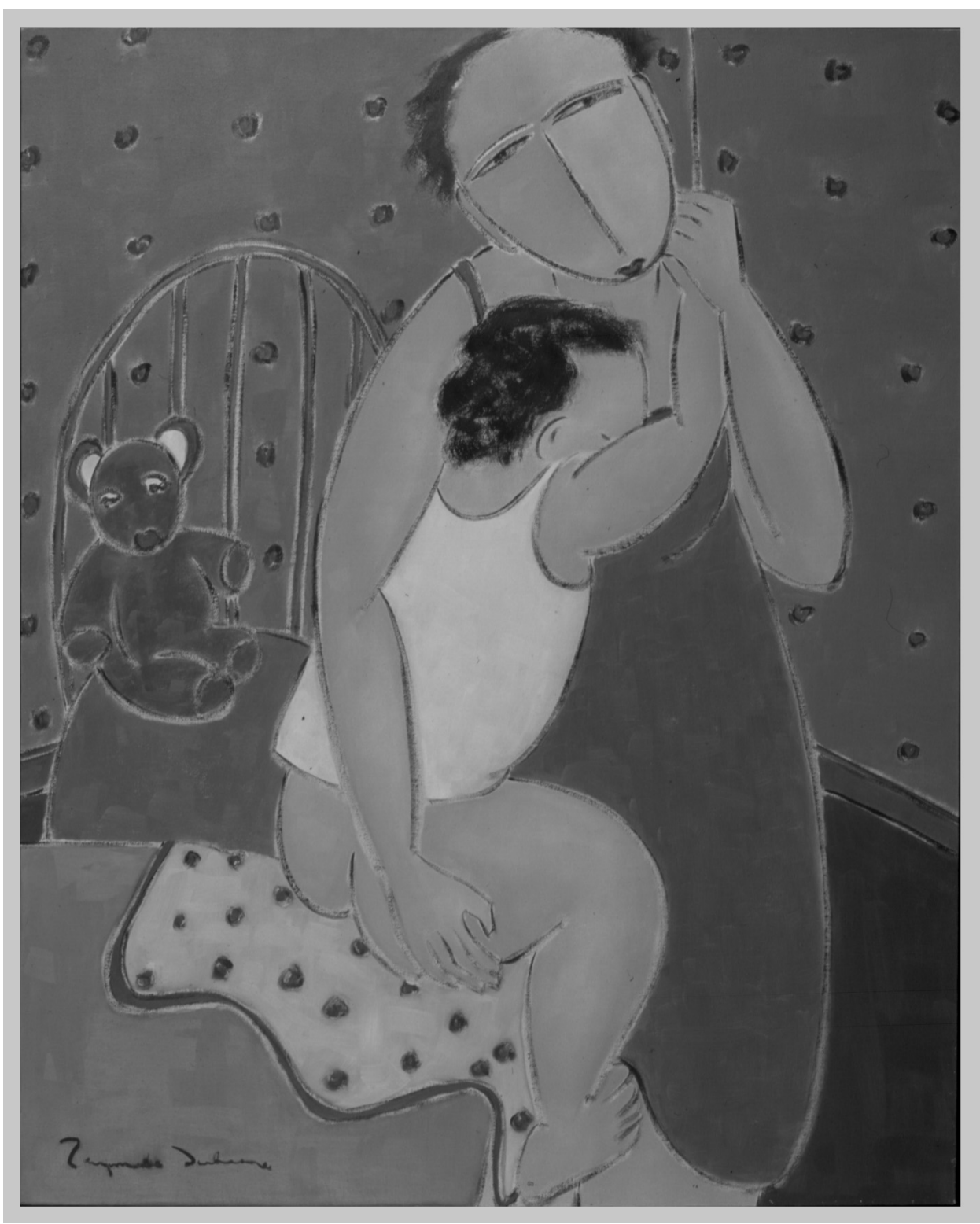

règles de bonne pratique médicale et de bon emploi de la médication ont été revues par le Collège et ont été signifiées aux médecins. Ces règles sont celles de la bonne prise en charge des patients, de la bonne connaissance des molécules et de la bonne tenue des dossiers. Elles sont exactement les mêmes que pour tout autre champ d'activité médicale.

Il y a donc méconnaissance, tant au niveau professoral que de l'enseignement de premier cycle universitaire, de la maladie douloureuse chronique et des médicaments pouvant la soulager. Cependant, nous croyons que le futur ne sera heureusement pas le reflet du passé, car des solutions sont apparues et ne cessent d'être exploitées.

\section{LES SOINS PALLIATIFS}

En Angleterre, durant les années 1970, est survenue une prise de conscience de l'importance de soigner la douleur chronique cancéreuse. Cette redécouverte, dans les hospices anglais, d'une partie importante de l'essence même de la médecine s'est propagée par la suite aux autres continents. Les cancéreux hospitalisés dans ces institutions ont pu recommencer à bénéficier de l'emploi de la morphine et de ses dérivés pour soulager leurs douleurs réfractaires. Il peut paraître inconcevable que cette classe de médicaments analgésiques connue depuis des millénaires pour ses vertus thérapeutiques ait eu à disparaître de la pharmacopée pour des raisons sociales et judiciaires. Cette redécouverte et ce nouveau départ se sont en quelque sorte faits par la porte arrière et sans tambour ni trompette. En suivant un raisonnement par étapes, on pourrait résumer comme suit: "Qui dit cancer et cancéreux, dit espérance de vie très raccourcie (du moins en 1970) et donc permission d'employer ces médicaments pour procurer une fin de vie acceptable sous ton aile

\section{s'adoucit le difficile}

avec le moins de douleur possible et avec une crainte de dépendance psychologique qui n'est plus un enjeu pour la société, la police et les médecins. » Il nous fallait bien repartir de quelque part.

Beaucoup de chemin a été parcouru depuis ces années de reconnaissance contemporaine des bienfaits des opioïdes dans le soulagement de la douleur. Beaucoup de recherches fondamentales et de résultats continuent d'affluer au regard de la pharmacocinétique de ces médicaments, de leur place dans la physiologie normale du corps humain et des risques réels peu élevés de toxicomanie lorsque employés pour soulager la douleur et non à des fins récréatives.

Les progrès sont palpables et perçus; le ministère de la Santé et des Services sociaux du Québec, par la voix du ministre Philippe Couillard, a fait part en mai 2004 au dernier congrès du Réseau québécois de soins palliatifs (RQSP) de sa volonté d'encourager le développement des services de soins palliatifs sur une plus grande échelle. De tels vœux ont déjà été exprimés au cours des 15 dernières années sans que des actions concrètes ne viennent appuyer le discours politique; il faut espérer que cette fois-ci soit la bonne.

Au plan canadien, les instances universitaires sont conscientes du besoin de former nos médecins de demain à l'art et à la science du soulagement de la douleur. Encore là, c'est la philosophie palliative qui prévaut. Se tenait à Montréal en septembre 2004, dans le cadre du XV Congrès international sur les soins aux malades en phase terminale, une réunion de travail des représentants des universités canadiennes pour faire le point sur l'enseignement des soins palliatifs; les participants ont exprimé le désir de déposer rapidement un programme national de formation universitaire de premier et de deuxième cycles. Les travaux sont déjà avancés et, au Québec, le Réseau universitaire québécois en soins palliatifs (RUQSP) finalise ses propres recommandations afin de les acheminer à chaque responsable de programme universitaire pour décision locale. 
L'avantage de structurer un programme intégré de premier cycle universitaire est énorme. Il permettrait à tous les futurs médecins de prendre contact de façon théorique et clinique avec les soins palliatifs avant que ne s'opère la division entre médecine familiale et spécialisée. Dans ce programme, une large base est accordée au soulagement de la douleur, à l'empathie tant envers le malade qu'envers sa famille, au dialogue avec ces malades et à la communication avec ces familles. Déjà il existe des stages obligatoires en soins palliatifs pour les médecins-résidents en médecine familiale. Ces stages sont offerts après l'obtention du diplôme universitaire et ne reposent pas sur des assises théoriques unifiées. De gros efforts sont aussi déployés afin de rendre de tels stages obligatoires pour les médecinsrésidents en spécialité.

\section{LA DOULEUR CHRONIQUE} NON CANCÉREUSE (DCNC)

Mission accomplie? Pas vraiment! En effet, il existe une autre population de malades souffrants qui ne bénéficient pas encore nécessairement de l'assouplissement par rapport aux préjugés mentionnés ci-dessus. Ces gens, souvent jeunes adultes, sont porteurs de maladies chroniques non cancéreuses post-traumatiques ou dégénératives. Très souvent, ce sont des accidentés de la route ou du travail. Aux notions erronées dont nous avons parlé s'ajoutent des préjugés professionnels. Ces malades sont souvent perçus et traités comme s'ils étaient coupables de ne pas pouvoir retourner à l'ouvrage et ils ont à prouver leur innocence à travers des dédales administratifs et de multiples expertises médicales. Une majorité d'experts médicaux n'acceptent pas les douleurs chroniques comme invalidantes. Ils ont tendance à ne considérer que l'accident déclencheur avec les séquelles physiques tout en niant ce qu'ils ne peuvent évaluer avec une image radiologique ou une manœuvre clinique, c'est-à-dire la douleur.

Or ces malades avec douleur chronique non cancéreuse sont des gens qui étaient actifs dans leur profession et qui tout d'un coup voient leur univers basculer. Ces malades sont handicapés et ne peuvent retrouver une capacité physique adéquate qui leur permettrait de redonner un plein rendement au travail, ce qui les place dans une situation difficile vis-à-vis de leur patron. En outre, lorsque la vie au travail est difficile, c'est souvent la vie familiale, la vie matrimoniale et la vie économique qui sont en suspens et qui finissent par être perturbées. La douleur empêche très souvent un sommeil réparateur; la fatigue anormale qui s'ensuit diminue encore plus l'énergie disponible pour les activités de la vie quotidienne. Une boucle infernale se met en place: fatigue physique et morale sont entremêlées, aboutissant à un découragement au mieux ou à une profonde dépression avec auto-dévalorisation et perte de l'image positive de soi, ou pire, ce sont des idées suicidaires qui apparaissent. Cette douleur globale et totale devient alors synonyme de "souffrance».

Pendant tout ce temps, ces personnes doivent subir les frustrations et parfois les comportements antipathiques des personnes travaillant pour la CSSTQ et la SAAQ; ils sont souvent harcelés avec des formulaires à faire remplir et des menaces d'interruption de paiement de leurs prestations s'ils ne fournissent pas tel ou tel papier. Ceux qui résistent doivent le faire à coup de frais judiciaires élevés et très souvent au prix de leur santé mentale; ce sont finalement les tribunaux qui trancheront au bout de plusieurs années.

Nos patients n'ont tout simplement pas le calme, la sérénité et la compréhension voulue pour cicatriser leurs lésions physiques et émotionnelles. Les multiples frustrations vécues et l'impuissance à faire accepter leurs douleurs ne font qu'ajouter des éléments négatifs qui modulent à la hausse les douleurs. En décrivant cette situation, je peux facilement mettre sur mes mots le visage des nombreux malades qui ont vécu les mêmes expériences d'irrespect de la part de personnes en poste et de mise en doute de leur crédibilité, en plus du peu d'empathie des médecins-experts. Ces derniers ne sont jamais des médecins œuvrant en clinique de douleur et ils s'attardent presque exclusivement à la lésion primaire, en laissant de côté la nouvelle maladie qu'est la douleur chronique. C'est comme si ces personnes et ces organismes avaient fait le choix de traiter les malades comme des fraudeurs et que ceux-ci devaient prouver leur innocence. C'est le contraire de notre système de justice. Pourtant une majorité de nos patients souffrant de douleur chronique n'a pas grands gains secondaires à tirer de la maladie puisqu'ils ont souvent perdu beaucoup plus dans leur vie professionnelle, familiale et matrimoniale que ce qu'ils réclament. Incompréhension de leur douleur et douleur de l'incompréhension sont souvent le recto et le verso du même drame humain chez ces gens. Chaque histoire est différente tout en se ressemblant dans la démarche, la colère, la frustration vécues par les acteurs qui y prennent part.

En somme, le sujet de la douleur chronique n'est pas un sujet qui a la cote auprès du personnel médical. Pourtant, les personnes qui souffrent de douleur chronique sont des malades particuliers qui demandent une attention particulière. La formation des médecins est déficiente au regard de la douleur et de ses mécanismes fondamentaux comme elle est déficiente dans les connaissances des traitements. Ces malades chroniques se heurtent à bien des embûches tant administratives qu'humaines. Le pire écueil étant l'incompréhension et les frustrations qui en découlent tant personnelles, professionnelles que familiales. Constat noir et sans espoir? Pas encore puisque les gouvernements et les universités semblent vouloir s'intéresser au sujet. Par la suite, il faudra y intéresser les médecins. Combien de temps cela prendra-t-il? Probablement beaucoup puisque former un médecin au point de vue scientifique demande entre 7 et 10 ans, alors que former un médecin au point de vue humain peut être beaucoup plus long!

\section{Bibliographie}

CHABAL, C. et M.K. ERJAVEC (1997). «Prescription opioid abuse in chronic pain patients clinical criteria, incidence, and predictors», Clinical Journal of Pain, 13(2), p. 150-155.

CHAPMAN, C.R. (1997). "Émotion et douleur», dans M. BRASSEUR, M. CHAUVIN et G. GUILBAUD (dir.), Douleurs, bases fondamentales, pharmacologie, douleurs aiguës, douleurs chroniques, thérapeutiques, Paris, Maloine.

FONBERG, E. (1986). "Amygdala, emotions, motivation and depressive states », dans R. PLUTCHICK et H. KELLERMAN (dir.), Emotion: Theory, Research and Experience (vol. 3), New York, Academic Press.

MELZACK, R. et K.L. CASEY (1968). «Sensory, motivational and central determinants of pain: a new conceptual model », dans D. KENSHAHO (dir.), The Skin Senses, Springfield, Illinois, C.C. Thomas.

MELZACK, R. et P. WALL (1989). Le défi de la douleur, Paris, Edisem Inc.

MERSKEY, H. et D.N. BOGDUK (dir.) (1994). Classification of Chronic Pain, Second Edition, IASP Task Force on Taxonomy, Seattle, IASP Press.

ROBERT, P. (1991). Dictionnaire alphabétique et analogique de la langue française, Paris.

PLUTCHICK, R.A. (1980). «General psychoevolutionary theory of emotion", dans R. PLUTCHICK et H. KELLERMAN (dir.), Emotion: Theory, Research and Experience (vol. 1), New York, Academic Press.

ROBINSON, R.C., R.J. GATCHEL, P. POLANTIN, M. DESCHNER, C. NOE et N. GAJRAJ (2001). "Screening for problematic opioid use», Clinical Journal of Pain, 17(3), p. 220- 228.

ROLLS, E.T. (1986). "Neural systems involved in emotion in primates", dans R. PLUTCHICK et H. KELLERMAN (dir.), Emotion: Theory, Research and Experience (vol. 3), New York, Academic Press.

SCARRY, E. (1985). The Body in Pain: The Making and Unmaking of the World, New York, Oxford University Press. 